\title{
Editorial
}

\section{RETROSPECTIVE ON THE FORUM}

\author{
BRIAN SEGAL
}

The National Forum on Post-Secondary Education raised many expectations about the future of post-secondary education (PSE) in Canada - expectations about the place of post-secondary education on the public policy agenda, about accessibility, about greater co-operation between federal and provincial governments, about the need for more research on post-secondary education, about enhanced funding for teaching and research, and about more collaboration between our colleges and universities. Two and half years later, although some of these expectations have been met, the fundamental problems confronting our universities and colleges remain.

The forum highlighted the importance of post-secondary education, raised public concerns about the subject and, by featuring participation from the many vitally interested sectors of society, it created a climate favourable to co-operation and constructive exchange. In the period since the forum, however, postsecondary education has not succeeded in a substantive way in occupying a primary place on the public policy agenda in Canada. Nor is there any definitive evidence that Canadians have come to recognize the urgency of the problems faced by our universities and colleges, or that the public is increasing its pressure on both levels of government to take those policy and funding actions necessary to strengthen the quality and diversity of our institutions.

This is not to suggest that the last two and half years have been devoid of positive initiatives. Although it is not possible to empirically correlate all of these initiatives with the forum, some appear to have had their genesis there. Certainly, a number of important intergovernmental actions relate directly to the forum. The establishment by the Council of Ministers of Education for Canada (CMEC) of a Ministerial Post-Secondary Committee came on the heels of the forum. Previously, the Council of Ministers of Education for Canada had been preoccupied to a significant degree with elementary and secondary education. In June 1988 and again in September 1989, this new committee met with representatives of Association of Universities and Colleges of Canada (AUCC), Association of Community Colleges of Canada (ACCC), Canadian Association of University Teachers (CAUT) and the Canadian Federation of Students (CFS) and confirmed their resolve to address the priority needs identified at the forum and to collaborate in the interest of post-secondary education. To date, no noticeable evidence exists that this resolve has resulted in concrete actions to improve the post-secondary 
education situation. Although in some provinces the political rhetoric about the importance of higher education has amplified, the link between rhetoric and action remains difficult to discern.

Some other developments include the establishment by the Council of Ministers of Education for Canada and Statistics Canada of a Canadian Education Statistics Council. In September 1989, the Secretary of State announced amendments resulting from its review of the Canada Student Loans Program. These changes allow greater flexibility in setting repayment terms, remedy certain administrative problems leading to "technical default" and introduce borrowing controls by linking assistance to the student's level of study.

In the area of research, the $\$ 1.3$-billion allocation by the federal government for new science and technology initiatives over a five-year period was announced at the forum of the National Conference on Technology and Innovation chaired by the prime minister. Under this program, the granting councils will receive an additional $\$ 200$ million to their base budgets over five years, the National Centres for Excellence program was created with an allocation of $\$ 240$ million, and the Canada Scholarship Program was announced with an allocation of $\$ 80$ million.

International student enrolments at the post-secondary education level increased by about eight per cent in 1988/89, the first increase since 1983/84. The Canadian International Development Agency (CIDA) has doubled the number of students and trainees assisted over a five-year period, and Canada Employment and Immigration Centre (CEIC) has provided greater flexibility for international students and their spouses. In September 1989, the Council of Ministers of Education for Canada announced the establishment of a national commission for the international recognition of students, which is jointly funded by the provinces and the Secretary of State.

During the first Minister's Conference in November 1989, the prime minister proposed a collective National Task Force on Human Resource Development for the Year 2000.

In Ontario, the minister of colleges and universities launched Vision 2000, a comprehensive review of the role, function and structure of the community college system, a move that was heavily influenced by the discussions at the forum. Specifically, the discussion on human resource requirements, accessibility and the relationship between colleges and universities helped to shape the terms of reference and broadly based participation structure of Vision 2000.

In British Columbia, the expansion of accessibility through the creation of additional degree-granting institutions and the general improvement in postsecondary education funding may also have had their roots in forum decisions.

Across the country, there has been a significant increase in post-secondary education enrolments, particularly in the universities, although generally inadequate resources have meant a continual trade-off between accessibility and quality, with quality suffering heavily under the burden of a large increase in the number of students and inadequate resources to meet the demand. 
Of these initiatives, very few can be characterized as having a significant and timely impact on solving critical problems within post-secondary education. Although the \$1.3-billion federal allocation for science and technology initiative is having a positive result, it must be coupled with the cap on the growth of Established Program Funding (EPF) transfer payments and the lack of availability of base budgets to support the infrastructure necessary for these initiatives.

Many of the initiatives, although necessary for the long term, are essentially process activities that do little to solve current and anticipated problems. The prime minister's call for another national task force may continue to focus national attention on higher education, but will do little to address the urgent issues currently before us.

It is not clear to me or many others why further study is required. The problems are well known and documented, the solutions are quite clear, and the need for immediate action far outstrips the need for further study.

And so, disappointingly, many of the expectations of the forum have not been met. Perhaps over time, when Canadians come to realize that the real opportunities for their children have been seriously minimized because of government inaction, spurred on in large measure by our own apathy, we will demand that post-secondary education policy and funding truly become a national priority. Only then will our governments realize that their future success is tied to the attention and resources they allocate to post-secondary education.

\section{BILAN RÉTROSPECTIF DU COLLOQUE}

\section{BRIAN SEGAL}

Le Colloque national sur l'enseignement postsecondaire a mis en lumière les nombreuses exigences qu'implique l'avenir de cet enseignement au Canada; parmi celles-ci, il y a le rang qu'il occupe parmi les préoccupations des politiciens, son degré d'accessibilité, les conditions d'une meilleure coopération entre les gouvernements fédéral et provinciaux, les recherches qu'il faut mener dans ce domaine, l'augmentation du financement à accorder à l'enseignement et à la recherche et l'intensification de la collaboration entre les collèges et les universités. Après deux ans et demi, même si l'on a répondu à certaines de ces exigences, les problèmes les plus graves auxquels nos universités et collèges doivent faire face sont toujours là.

Le colloque a souligné l'importance de l'enseignement postsecondaire, provoqué l'inquiétude du public sur cette question et, en appelant la participation d'un grand nombre de secteurs directement concernés par lui, créé un bon climat d'échange et de coopération. Depuis ce temps toutefois, l'enseignement postse- 
condaire n’a pas vraiment réussi à gagner une place de premier plan parmi les préoccupations politiques prioritaires du Canada. Il n'y a pas non plus de preuve flagrante que les Canadiens reconnaissent enfin l'urgence des problèmes qui se posent aux universités et collèges ni que le public fasse davantage pression sur les deux paliers de gouvernement pour qu'ils prennent les mesures politiques et financières nécessaires à la mise en pratique d'un enseignement plus varié et de meilleure qualité au sein de nos établissements.

Nous ne voulons certes pas dire que ces deux dernières années n'ont vu l'introduction d'aucune action concrète. Quoiqu'on ne puisse pas prouver par des faits qu'il existe une corrélation entre ces actions et le colloque, il est permis de croire que certaines d'entre elles ont vu le jour à l'occasion de ce dernier. Sans doute d'importantes mesures prises entre les divers paliers de gouvernement sont-elles aussi directement issues du colloque. Ainsi, la création par le Conseil des ministres de l'Éducation du Canada (CMEC) d'un Comité ministériel sur l'enseignement postsecondaire l'a suivi de près. Auparavant le CMEC se préoccupait surtout de l'enseignement élémentaire ou secondaire. En juin 1988 et en septembre 1989, le nouveau comité s'est réuni avec des représentants de l'Association des universités et collèges du Canada (AUCC), de l'Association des collèges communautaires du Canada (ACCC), de l'Association canadienne des professeurs d'université (ACPU) et de la Fédération canadienne des étudiants (FCE); à cette occasion, le comité a réaffirmé sa volonté de prendre des mesures qui répondent aux besoins primordiaux que l'on avait pu déceler au cours du colloque et de travailler en collaboration avec ces organismes dans l'intérêt de l'enseignement postsecondaire. Jusqu'à présent il n'existe pas de preuve évidente que cette volonté ait abouti à des mesures concrètes visant à améliorer la situation à ce niveau de l'enseignement. Même si, dans certaines provinces, nous avons pu constater que la rhétorique politique accordait une plus grande place à l'importance de l'enseignement supérieur, il est encore difficile de voir un lien entre ces paroles et la mise en oeuvre de mesures concrètes.

Parmi les autres conséquences du colloque, il faut mentionner la création par le Conseil des ministres de l'Éducation du Canada et Statistique Canada conjointement d'un Conseil canadien des statistiques en éducation. En septembre 1989, le Secrétariat d'État a annoncé des amendements qui faisaient suite à une étude sur le Programme canadien de prêts aux étudiants. Ces modifications introduiront des conditions de remboursement plus flexibles, remédieront à certains problèmes d'ordre administratif susceptibles d'engendrer des "défauts techniques", et créeront un système de contrôle de l'emprunt liant le montant d'aide au niveau d'étude de l'étudiant.

Dans le domaine de la recherche, le premier ministre, au cours de la Conférence nationale sur la technologie et l'innovation qu'il présidait, a annoncé au colloque que le gouvernement fédéral allouerait 1,3 milliard de dollars sur cinq ans à des projets portant sur les sciences et les techniques nouvelles. Les conseils dispensateurs recevront en outre, en vertu de ce programme, une somme additionnelle de 200 millions de dollars à intégrer à leur comptabilité publique sur 
l'ensemble de cette période tandis que l'on mettrait le programme des centres nationaux d'excellence à exécution grâce à une allocation de 240 millions de dollars et que l'on créerait le programme Bourses Canada à l'aide d'une autre allocation de 80 millions.

Les inscriptions d'étudiants étrangers dans un établissement d'enseignement postsecondaire ont augmenté d'environ huit pour cent en 1988-1989: c'est la première fois qu'elles augmentent depuis 1983-1984. Pendant ces cinq dernières années, le nombre d'étudiants et de stagiaires aidés par l'Agence canadienne de développement international a doublé tandis que la Commission de l'Emploi et de l'Immigration faisait preuve de plus de flexibilité à l'égard des étudiants étrangers et de leurs épouses. En septembre 1989, le Conseil des ministres de l'Éducation du Canada a annoncé la création d'une commission nationale pour la reconnaissance internationale des étudiants financée à la fois par la province intéressée et par le Secrétariat d'État.

Au cours de la conférence des premiers ministres qui s'est tenue en novembre 1989, le premier ministre du Canada a proposé la création collective d'un groupe de travail national sur la mise en valeur des ressouces humaines en vue de l'an 2000 .

En Ontario, le ministre responsable des collèges et des universités a lancé son programme «Vision 2000 », programme qui passe en revue de façon complète le rôle, la fonction et la structure de l'ensemble des collèges communautaires: une lclle initiative a de toute évidence été fortement influencée par des discussions du colloque, et particulièrement par celles sur les besoins en ressources humaines des collèges, leur degré d'accessibilité et les relations qu'ils entretiennent avec les universités; toutes ces discussions ont permis d'établir les paramètres de «Vision $20(0)$ » à partir d'une représentation fidèle des divers intérêts concernés.

En Colombie-Britannique, on a rendu l'enseignement postsecondaire plus accessible en autorisant davantage d'établissements à délivrer des diplômes et en organisant le financement de façon plus adéquate: ces mesures pourraient bien être nées elles aussi au sein du colloque.

Partout au pays, il y a eu un regain important des inscriptions au niveau postsecondaire, notamment dans les universités; toutefois l'insuffisance des ressources a fait naître un déséquilibre toujours plus sensible entre l'accessibilité dans les établissements et la qualité de l'enscignement offert, cette dernière souffrant beaucoup de la charge que représente une forte augmentation du nombre d'étudiants lorsque les ressources disponsibles pour répondre à la demande sont insulfisantes.

Parmi ces mesures, bien peu semblent pouvoir résoudre, de façon importante et définitive, les problèmes critiques qui se posent à l'enseignement postsecondaire. Meme si l'allocation d'1,3 milliard du gouvernement fédéral produit déjà des résultats encourageants, il faudra qu'en plus elle s'associe à un taux plafond de croissance des paiements de transfert et tienne compte du manque de disponibilité de la comptabilité publique pour le financement de la mise en oeuvre des mesures prévies. 
Un grand nombre de ces mesures, sans doute nécessaires à long terme, sont essentiellement des moyens de traitement qui résolvent fort peu les problèmes actuels ou imminents. La proposition du premier ministre de créer un autre groupe de travail national aura peut-être pour effet de continuer à attirer l'attention du public sur l'enseignement supérieur, mais elle ne permettra pas de s'attaquer aux problèmes actuels les plus urgents.

Je ne comprends pas, et je ne suis pas le seul dans ce cas, pourquoi il faut mener une nouvelle étude. Nous connaissons bien les problèmes, de nombreux ouvrages en parlent; les solutions sont assez évidentes et le besoin d'agir vite dépasse de loin celui d'approfondir la recherche.

Alors, nous regrettons de le dire, bien des espoirs nés au colloque ont été déçus. Peut-être, avec le temps, les Canadiens comprendront-ils que les chances réelles de leurs enfants décroissent du fait de l'inaction du gouvernement, et que cette inaction résulte en grande partie de leur propre apathie; ils demanderont alors que l'enseignement postsecondaire devienne véritablement, tant du point de vue financier que politique, une priorité nationale. Ce n'est qu'à ce moment que nos gouvernements comprendront que leur succès dépend de l'attention et des ressources qu'ils sont prêts à accorder à cet enseignement. 